\title{
Measurement of the Free Spectral Range and the Side-Modes Suppression Ratio of a 9.3-GHz Single-Loop Opto-Electronic Oscillator by Fiber Path Selector
}

\author{
Mehmet Alp Ilgaz, Boštjan Batagelj \\ University of Ljubljana, Faculty of Electrical Engineering, \\ Ljubljana, Slovenia \\ mehmet.ilgaz@fe.uni-lj.si
}

\begin{abstract}
This paper describes measurements of the spurious modes of oscillation for a single-loop opto-electronic oscillator (OEO) at $9.3 \mathrm{GHz}$. The side modes of an OEO damage the oscillator's good spectral properties and therefore the behaviors of those unwanted modes need to be understandable in order to suppress them. Moreover, we review the state-of-the-art techniques for overcoming the side modes and suggest our measurement setup for effective testing of electrical bandpass filters.
\end{abstract}

Keywords - micro-wave oscillator, optical fiber, opto-electronic oscillator, side modes, side-modes suppression ratio.

\section{INTRODUCTION}

A high-frequency stable oscillator with a low phase noise is required for a variety of applications in medicine, astronomy, radar, wireless networking, etc. An optoelectronic oscillator (OEO) [1], [2] is one of the high-quality frequency oscillators that can generate signals in the micro-wave, and even the millimeter-wave range. In the micro-wave range it can be used for the distribution of the clock signal over a stabilized single optical fiber infrastructure [3], [4], while in the millimeter-wave range it is getting a lot of attention for fifth-generation mobile networks [5],[6].

Major research effort in the area of the OEO is currently taking place in minimization of it. Getting the low phase noise oscillator as a small integrated device will allow us using it in variety of mobile and driving applications. Today, we are in the way to monolithic integration of OEO [2], [7] and [8]. When we want to pack the OEO in small space, but still use external delay-line, the temperature and vibration sensitivity as well as electromagnetic interference are important [9]. The trade-off between achieving a low phase noise by long delay line and elimination of resonator side modes is always present. In this paper we describe measurements of the OEO spurious modes position and power that can be useful when external delay-line is used. Our non-integrated OEO has lower phase noise in comparison to the monolithic integration OEO since it has low Quality (Q) factor. The measurement results obtained in this work, help designers of a single-loop opto-electronic microwave oscillator to choose a proper electrical bandpass filter regarding with the necessary fiber length.

\section{REVIEW OF THE STATE OF THE ART}

In general, an OEO is composed of a laser diode, an opto-electronic modulator, an optical fiber (delay line), a photodetector, an electrical amplifier and filter, and other optical and electrical components such as a directional coupler [2]. In order to 
produce oscillation, the OEO should have Barkhausan conditions (gain and phase shift). In the OEO the optical Q-factor is directly proportional to the optical length of the delay line. In other words, if the length of the optical fiber is increased, the Qfactor is increased. However, by increasing the fiber length, the free spectral range (FSR) between the oscillation modes becomes smaller. The FSR can be defined as

$$
F S R=\frac{c_{0}}{n_{g} \cdot L}
$$

where $c_{0}$ is the speed of light, $n_{\mathrm{g}}$ is the group index and $L$ is the length of the cavity. As is clear from Eq.1, the FSR is inversely proportional to the optical fiber length. For example, with $1 \mathrm{~km}$ of optical fiber the FSR is around $200 \mathrm{kHz}$, but, on the other hand, it is $13.4 \mathrm{kHz}$ with a $15-\mathrm{km}$ OEO where $n_{\mathrm{g}}$ is 1.468 . An example of an OEO's output spectrum is shown in Fig. 1.

It is very well known, that with a longer optical fiber length, the oscillator modes become closer. Therefore, it is more difficult to suppress the side modes of the oscillation due to the bandwidth limit of the electrical bandpass filter used in the OEO's loop. The power of the side modes is based on the Q-factor of the electrical filter. At higher frequencies it is extremely demanding to manufacture a very narrowband bandpass filter. Therefore, the side modes are observed in the output spectrum of oscillator. In other words, side modes occurred due to not having ideal filter in the oscillator loop.

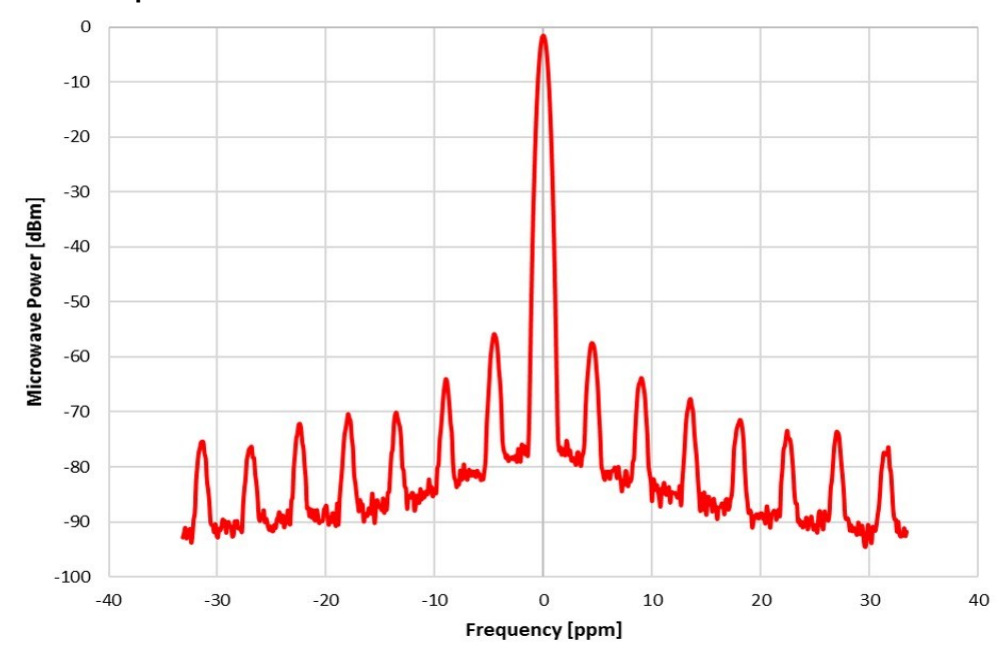

Fig. 1. Example of an optical spectrum at the output of a 9.3-GHz OEO [5].

As mentioned previously in the text, the length of the optical fiber with the modes relation is shown in Fig. 2. If the length of the fiber is increased, the FSR becomes smaller and the modes become closer together. Therefore, the electrical filter cannot suppress the modes because of the insufficient bandpass. 

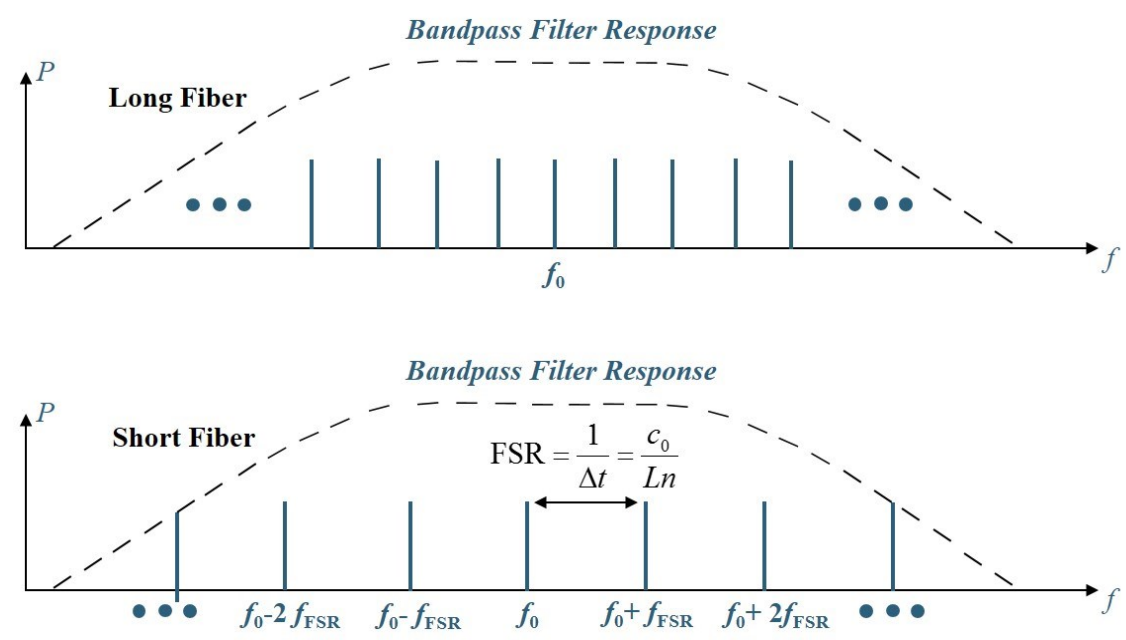

Fig. 2. Sketch of the OEO resonator modes for different lengths of the optical fiber.

The side-modes suppression ratio (SMSR) is defined as the proportion of the microwave or millimeter-wave power between the central oscillation frequency with the nearest higher-order spurious mode as shown in Fig. 3. The phase noise of the OEO depends on several concepts of the devices and phenomenon in the OEO's loop. Briefly, for a single-loop of OEO, the phase noise is based on laser relative intensity noise (RIN), laser frequency noise, shot noise from photodetector, amplifier noise and Rayleigh scattering on the optical fiber.

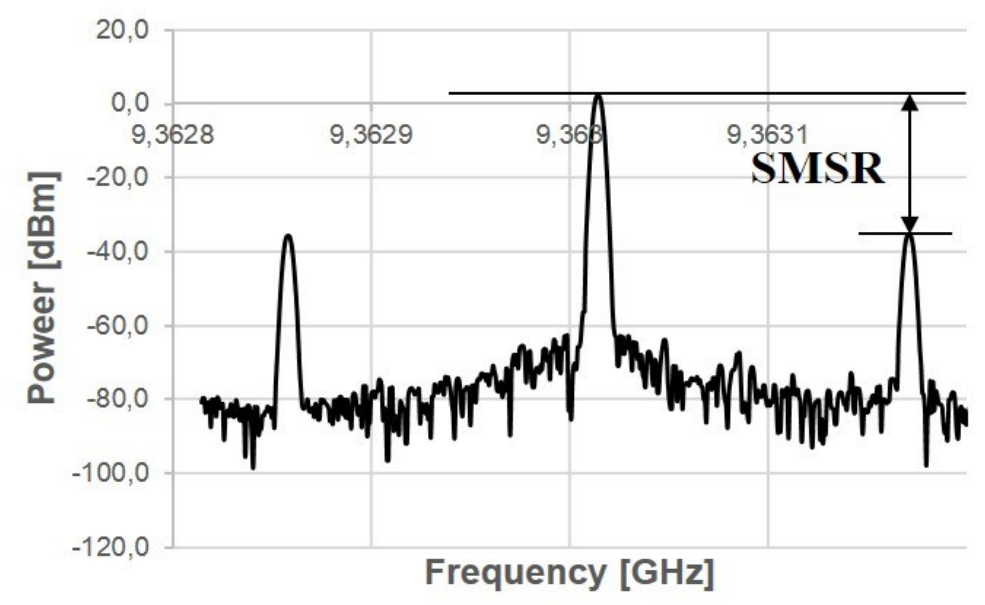

Fig. 3. Frequency spectrum of the OEO with the cavity filter at $3 \mathrm{GHz}$.

As briefly explained, for the multi-mode operation of an OEO there have been many solutions to suppress its side modes. One option is to implement a coupled OEO [10], where an additional feedback loop is used to beat with in-phase laser modes. Another possibility is to place another optical loop in the system, which is called a dual-loop OEO [11], where the long-loop contributes to the low phase noise, while the short-loop eliminates spurious modes. If the placement involves more than one additional optical loop it is called a multi-loop OEO [12]-[13]. Recently, a novel method was introduced in [14], i.e., to use a hybrid bandpass filter in an OEO's loop at $30 \mathrm{GHz}$ and have a $\mathrm{Q}$ of 30,000 . With this implementation, a SMSR of $83 \mathrm{~dB}$ can be achieved. There is also another route, called the Quality multiplier, which can decrease the bandwidth of the cavity filter of the OEO [15]. With this technique the 
side modes are suppressed by $20 \mathrm{~dB}$. There is also the possibility to use a photonic filter [16] instead of an electrical filter to suppress the side modes. Finally, there is the injection-locking method, which can be used to suppress the side modes [17]. With this technique two OEOs are injection locked and so no side modes are observed. A brief overview of possible side-modes suppression techniques shows us that we still do not have appropriate method. In order to have effective solution the behavior of those unwanted side modes need to be clear to designers of electrical bandpass filter. Our experimental setup can be used for the verification of designed electrical bandpass filters.

\section{PROPOSED MEAsUREMENT SETUP}

For the experimental verification of our electrical bandpass filter, we built a singleloop OEO with fiber path selector. An electrical bandpass filter is a microstrip filter with a bandwidth of $100 \mathrm{MHz}$ at a central frequency of $9.3 \mathrm{GHz}$. In the experimental work, the length of the optical delay line is changed from $1.25 \mathrm{~km}$ to $8.75 \mathrm{~km}$ with an increment of $1.25 \mathrm{~km}$. We used a standard single-mode fiber G.652D.

The aim of this proposed measurement setup (Fig. 4) is to show how the FSR between the modes changed when we alter the optical fiber delay line and to enable effective measurement of the electrical part of a single-loop OEO. The optical paths are changed and combined with an optical fiber path selector.

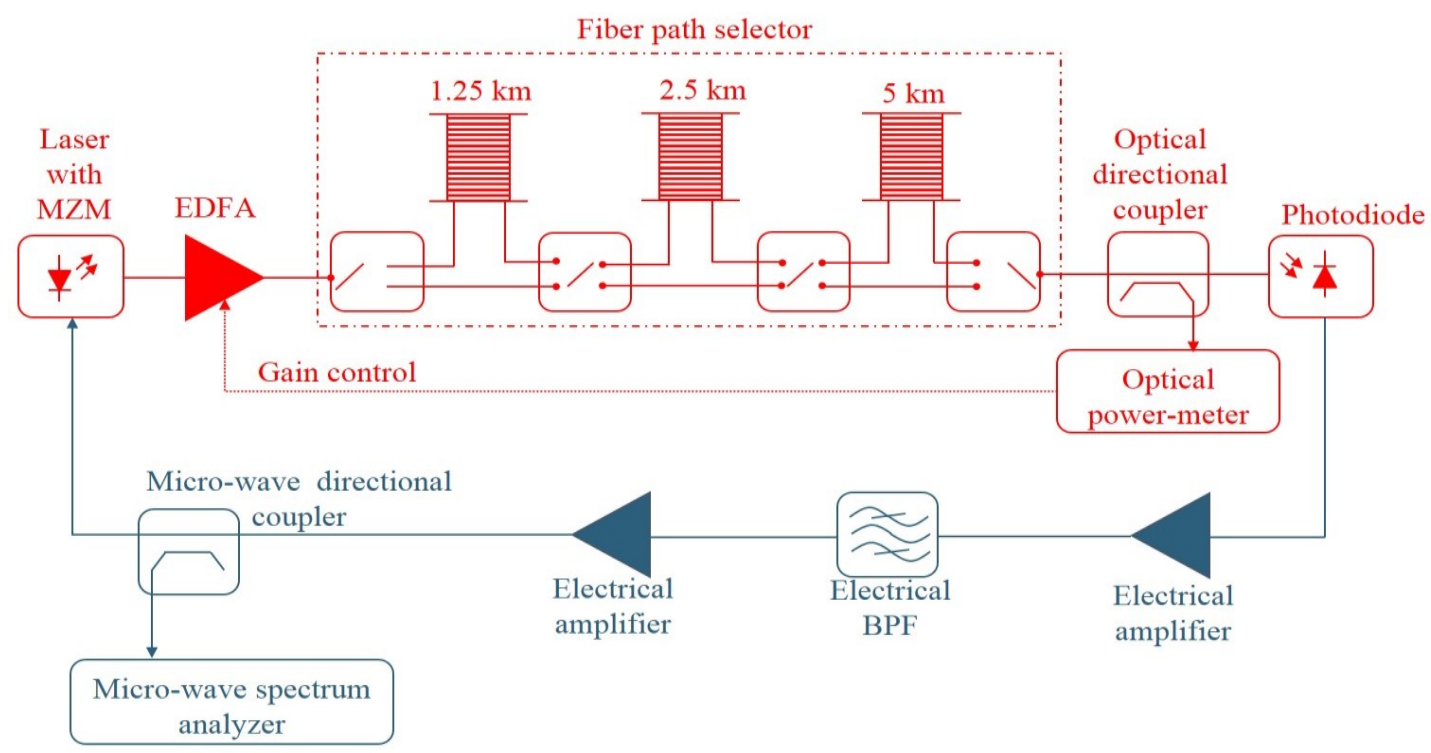

Fig. 4. Diagram of the experimental setup to measure the FSR and SMSR. BPF is an electrical bandpass filter.

The experimental setup consists of a single-loop OEO with an electrical bandpass filter and an electrical and optical amplifier. A binary III-V semiconductor InP-based Mach Zehnder Modulator (MZM) with integrated distributed feedback (DFB) laser is used to generate optical signal. The Mach Zehnder amplitude modulator is biased in quadrature point, while the laser is temperature-stabilized. There is a fiber path selector device that allows the user to change the optical paths rapidly. The insertion loss (IL) of this device is around $6 \mathrm{~dB}$. An erbium-doped fiber amplifier (EDFA) is used to compensate for the optical losses. The electrical amplifiers are used to compensate for the conversion loss (optical to electrical) and the IL of the electrical 
bandpass filter. An optical power meter is used in a feedback to control the EDFA when the optical length is selected with the fiber path selector. The purpose of a gain control is to tune the EDFA's pump laser to achieve always the same optical power at the OEO's photodiode. The gain control adjusts the power feedback seeded into the transmitter unit to obtain comparable results of electrical spectrum. A photograph of the measurement setup is shown in Fig. 5.

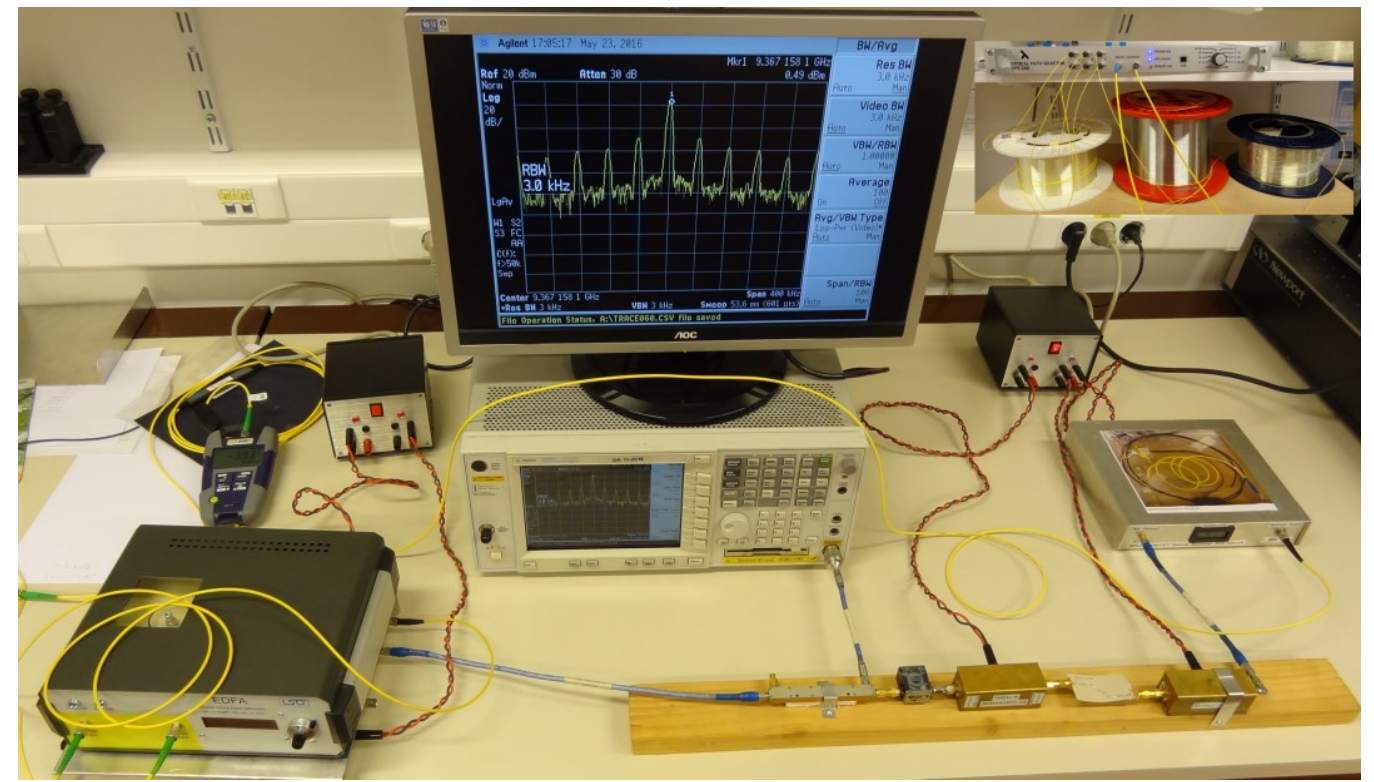

Fig. 5. Photograph of the measurement setup to measure the FSR and SMSR by fiber path selector.

The results of oscillator spectrum were obtained with an Agilent Spectrum Analyzer. On the right-top corner of the photograph is the optical fiber $(1.25-\mathrm{km}, 2.5-\mathrm{km}$ and 5 $\mathrm{km})$ connected by an optical fiber path selector.

The measurement results are shown in Table 1, Fig. 6 and Fig. 7. For each measurement fiber length, the amplification of the EDFA is adjusted to compensate for the fiber attenuation. This allows us to have the same power at the input of the photodiode and therefore the power measurement conditions stay the same, while the fiber length is selected to have a new value.

TABLE 1: MEASUREMENT RESULTS ON THE FSR AND SMSR.

\begin{tabular}{|c|c|c|}
\hline Fiber Length $[\mathrm{km}]$ & FSR $[\mathrm{kHz}]$ & SMSR [dB] \\
\hline 1.25 & 156.7 & $>30$ \\
\hline 2.5 & 80.2 & $>27$ \\
\hline 3.75 & 52.7 & $>28$ \\
\hline 5 & 40.7 & $>14$ \\
\hline 6.25 & 32.1 & $>12$ \\
\hline 7.5 & 26.8 & $>8$ \\
\hline 8.75 & 24.00 & $>7.5$ \\
\hline
\end{tabular}



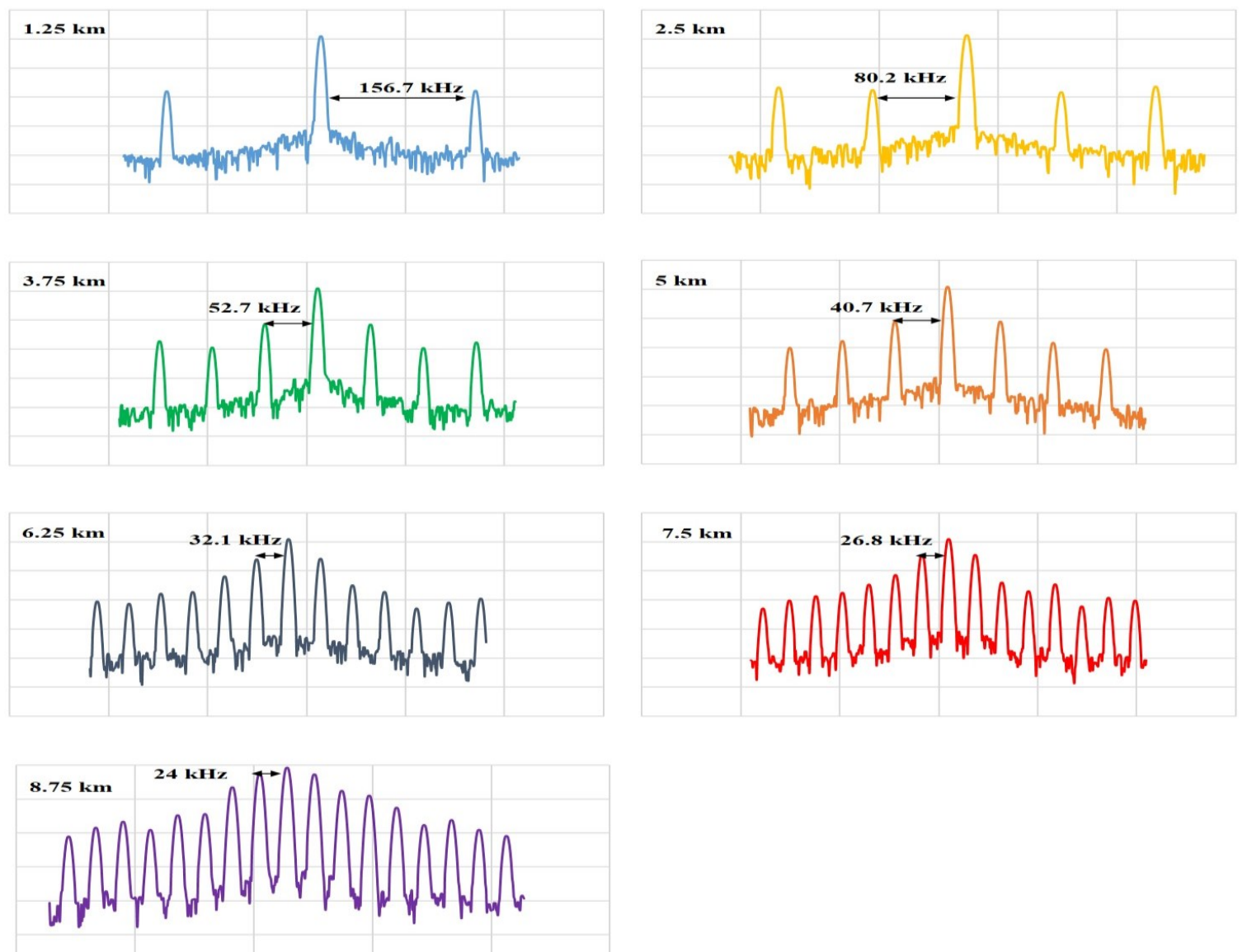

Fig. 6. Experimental results. The data is directly obtained from the spectrum analyzer. The data are used to measure the FSR and the SMSR.

Table 1 shows the high change (about $15 \mathrm{~dB}$ decrease) on SMSR when we change the fiber length from $3.75 \mathrm{~km}$ to $5 \mathrm{~km}$. We assume that the reason is related to the characteristics of the electrical filter of the OEO. Fig. 7 shows the experimental result and theoretical calculation on the FSR.

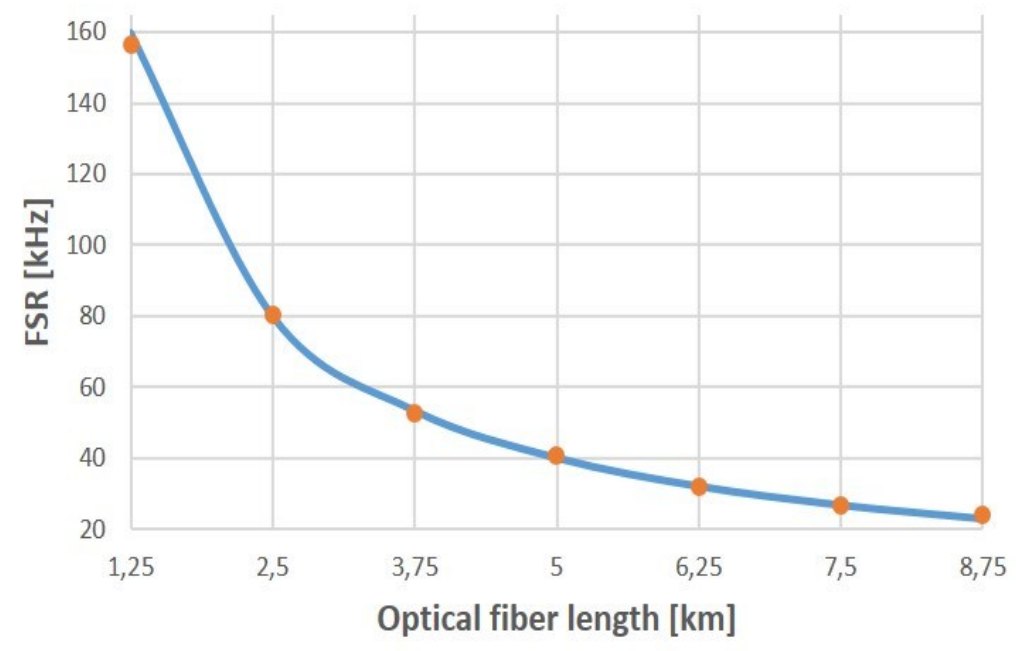

Fig. 7. FSR comparison between theoretical calculations and experimental results. The blue line shows the theoretical calculations and dots show the experimental results. 
As it is clearly seen from Fig. 7, the experimental results overlap with the theoretical calculations. Fig. 8 shows the experimental result on the SMSR with optical fiber length.

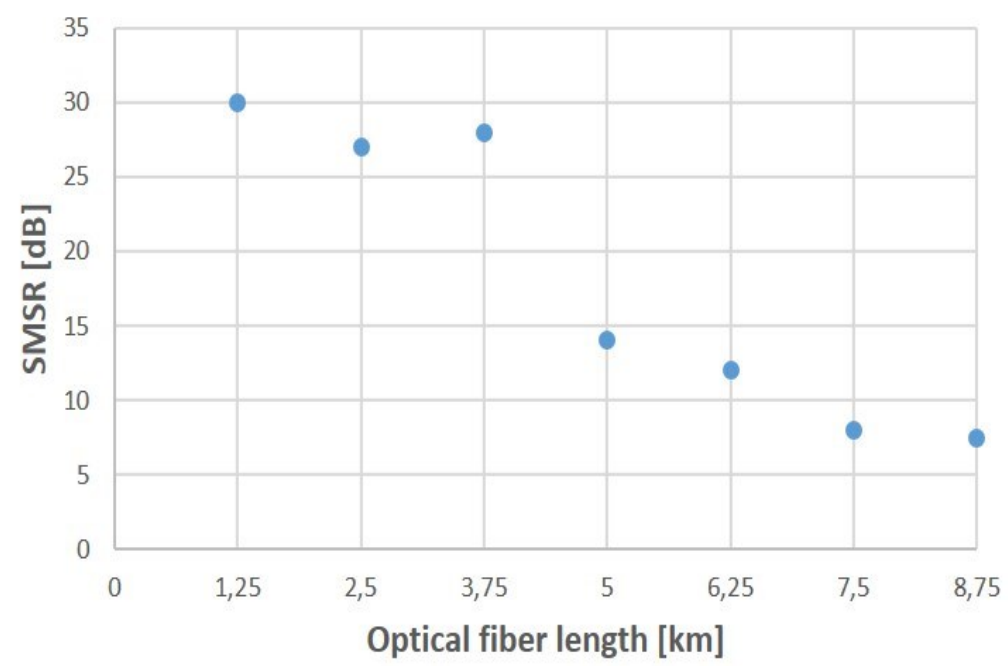

Fig. 8. SMSR change with the optical fiber length for a single-loop OEO.

Based on the results, proposed measurement setup supports the theory related to the FSR. Therefore, the optical fiber length should be taken into consideration when designing an OEO. In addition, from Fig. 8 it is clear that the SMSR is decreased with the increased fiber length of the OEO. This can be explained by the bandwidth of the electrical bandpass filter. When the optical fiber length is increased, the modes become closer to the main mode (where the electrical bandpass filter determines the oscillation frequency and it is not identical) so the modes are less suppressed compared to the shorter optical fiber lengths. Therefore, the power of the modes is increasing as the SMSR is decreased.

\section{CONCLUSION}

This paper briefly describes the methods used for an OEO to suppress the side modes of the oscillation. For the single-loop OEO we show how the FSR is changing with the optical delay line's length. There is a trade-off between the side modes and the phase noise. In other words, in order to decrease the phase noise of the OEO, the delay of the optical line should be increased. This can be done by increasing the optical fiber length. On the other hand, if the length of optical fiber is increased the FSR will be lowered that makes harder to suppress the side modes due to the insufficient bandwidth of the electrical filter. To overcome this trade off, one solution is to increase the $Q$-factor of the filter. Even though the length of the optical fiber is increased while improving the Q-factor of the filter, it could be possible to increase the phase noise. The reason in the increment of the phase noise in OEO with quality multiplier is that the Q-factor of the filter is increased by adding extra electrical circuit which adds electronic noise on the loop and increase the phase noise. As a conclusion, increasing the Q-factor of the filter should be thought clearly to avoid additional phase noise. We believe that our measurement setup for quantification of the FSR and the SMSR of a single-loop OEO by fiber path selector can help designers of band pass filters to evaluate side mode suppression rate faster. 


\section{ACKNOWLEDGMENT}

The authors would like to express their gratitude to the company InLambda BDT d.o.o. for the research equipment and devices. The work presented in this article was created within the FIWIN5G Innovative Training Network, which has received funding from the European Union's Horizon 2020 Research and Innovation Programme 2014-2018 under the Marie Skłodowska-Curie Action, grant agreement No.642355.

\section{REFERENCES}

[1] X.S. Yao, and L. Maleki, "High frequency optical subcarrier generator," in Electronics Letters, vol. 30, no. 18, pp. 1525-1526, Sep.1994.

[2] T. Hao, J. Tang, D. Domenech, W. Li, N. H. Zhu, J. Capmany, and M. Li, "Towards monolithic integration of OEOs: From systems to chips," in J. Lightw. Technol., vol. 36, no. 19, pp. 4565 - 4582, 2018.

[3] J. Tratnik, P. Lemut, U. Dragonja, B. Batagelj, and M. Vidmar, "Distribution of RFclock signal over single optical fiber with temperature-drift and vibration compensation," in MWP 2013, Alexandria, VA, 2013, pp. 162-165.

[4] J. Tratnik, L. Pavlovic, B. Batagelj, P. Lemut, P. Ritosa, M. Ferianis, and M. Vidmar, "Fiber length compensated transmission of $2998.01 \mathrm{MHz}$ RF signal with femtosecond precision," in Microwave and Optical Technology Letters, vol. 53, no. 7, pp. 1553-1555, Jul. 2011.

[5] M. A. Ilgaz, and B. Batagelj, "Preliminary idea for a converged fixed and mobile network infrastructure with $5 \mathrm{G}$ using Radio-over-Fiber technology and an OptoElectronic Oscillator in the millimeter-wave range," in ICTON 2016, Trento, 2016, pp. 1-4, Jul. 2016.

[6] M. A. Ilgaz, and B. Batagelj, "Opto-electronic oscillator in the mm-W range for $5 \mathrm{G}$ wireless and mobile networks: Design challenges and possible solutions," in ONDM 2017, Budapest, 2017, pp. 1-5, May 2017.

[7] J. Tang, T. Hao, W. Li, N. Zhu, and M. Li, "An integrated optoelectronic oscillator," in MWP 2017, Beijing, 2017, pp.1-4, Oct. 2017.

[8] X. S. Yao, L. Maleki, and V. Ilchenko, "Integrated opto-electronic oscillator having optical resonators," U.S. Patent 6873631, Mar. 2005.

[9] K. Mikitchuk, A. Chizh, and S. Malyshev, "Theoretical investigation of external influences on delay-line optoelectronic oscillator," in MWP 2015, Paphos, 2015, pp. 1-4, Oct. 2015.

[10]X.S. Yao, L. Davis, and L. Maleki, "Coupled optoelectronic oscillators for generating both RF signal and optical pulses," in J. Lightw. Technol., vol. 18, no. 1, pp. 73-78, Jan. 2000.

[11]J. Yang, Y.Jin-Long, W. Yao-Tian, Z. Li-Tai, and Y. En-Ze, "An Optical Domain Combined Dual-Loop Optoelectronic Oscillator," in IEEE Photon. Techn. Lett., pp. 807-812, June 2007.

[12]D. Eliyahu, and L. Maleki, "Low phase noise and spurious level in multi-loop opto-electronic oscillators," in IEEE International Frequency Control Symposium and PDA Exhibition Jointly with the 17th European Frequency and Time Forum, 2003. Proceedings of the 2003, 2003, pp. 405-410.

[13]T. Bánky, B. Horváth, and T. Berceli, "Optimum configuration of multiloop optoelectronic oscillators," in J. Optical Soc. of Am. B, vol. 23, no. 7, pp. 13711380, July 2006. 
[14]A. Liu, J. Liu, J. Dai, Y. Dai, F. Yin, J. Li, Y. Zhou, T. Zhang, and K. Xu, "Spurious Suppression in Millimeter-Wave OEO with a High-Q Optoelectronic Filter," in IEEE Phot. Techn. Lett., vol. 29, no. 19, pp. 1671-1674, Oct. 2017.

[15]L. Bogataj, M. Vidmar, and B. Batagelj, "Opto-electronic oscillator with quality multiplier," in IEEE Trans. Microw. Theory Techn., vol. 64, no. 2, pp. 663-668, Feb. 2016.

[16]M. Bagnell, J. Davila-Rodriguez, and P.J. Delfyett, "Millimeter-wave generation in an optoelectronic oscillator using an ultrahigh finesse etalon as a photonic filter," in J. Lightw. Technol., vol. 32, no. 6, pp. 1063-1067, Mar. 2014.

[17]W. Zhou, and G. Blasche, "Injection-locked dual opto-electronic oscillator with ultra-low phase noise and ultra-low spurious level," in IEEE Trans. Microw. Theory Techn., vol. 53, no. 3, pp. 929-933, Mar. 2005. 